\title{
Research Article \\ Study on Identification Method for Parameter Uncertainty Model of Aero Engine
}

\author{
Jie Bai $\mathbb{D}^{1,2}$ Shuai Liu $\mathbb{D}^{1},{ }^{1}$ and Wei Wang ${ }^{2}$ \\ ${ }^{1}$ School of Mechanical Engineering, Hebei University of Technology, Tianjin 300130, China \\ ${ }^{2}$ Key Laboratory for Civil Aircraft Airworthiness Certification Technology, Civil Aviation University of China, Tianjin 300300, China
}

Correspondence should be addressed to Shuai Liu; caucliushuai@163.com

Received 9 September 2019; Revised 12 November 2019; Accepted 22 November 2019; Published 2 December 2019

Academic Editor: Zhiguang Song

Copyright (C) 2019 Jie Bai et al. This is an open access article distributed under the Creative Commons Attribution License, which permits unrestricted use, distribution, and reproduction in any medium, provided the original work is properly cited.

\begin{abstract}
The linear model of an aero engine is effective in a small range of the neighborhood of equilibrium points. According to this problem, the identification method for the parameter uncertain linear model of the aero engine was proposed. The identification problem is solved by calculating nonlinear programming. Considering the parameter uncertainty of the model is the critical point of this research during the optimization process. A parameter uncertain model of an aero engine can be obtained, which has large use range. This method is used for DGEN380 aero engine. The two parameters, VDD and VE, are defined for describing error range. Compared with experimental data, the uncertain model of DGEN 380 can simulate the real state of DGEN380 within $1 \%$ error range when $\triangle \mathrm{PLA}<22 \%$. Compared with another conventional method of identification (recursive least squares), the parameter uncertain model, established by the method of this research, has a broad application area through parameter uncertainty of the model.
\end{abstract}

\section{Introduction}

The mathematical model describes the relationship among aero engine state variables and input variables through mathematical logic and mathematical language which is often used for the engineering design of engine control system and engine fault diagnosis widely [1]. The nonlinear mathematical model is hardly used for the engineering design of aero engine control system and fault diagnosis on account of the complicated structure, complex form, and highly nonlinear behavior which can describe the variation of each parameter in a full envelope range of aero engine [2]. There is an approximately linear correlation between every parameter of aero engine when the state of aero engine approaches the steady state point. The nonlinear mathematical model is transformed into a linear model by linearizing at the steady state point. The linear model is usually used for controller design. The function of this controller is steady control. The transient control process between two steady states is realized by interpolating the gain parameters, namely, gain scheduling which is a conventional method. Only in this way, can the control system of aero engine exert the control function in the full envelope [3].

The aero engine linear model has characteristics of having a simple form, fast calculation speed, and others [4], which are usually used in control system design and fault diagnosis [1]. However, the difference between the first order differential of engine state variables and the linear model slope increases as the distance between the engine state point and the steady state point increases, which narrows the application range of linear model and influences the application range of the controller and observer near the steady state point. If the application scope of the aero engine linear model is more than this limited range, the accuracy of the aero engine linear model is less than $90 \%$. The small application range is usually less than $10 \%$ of the neighborhood of equilibrium point in engineering.

The control problem and diagnosis problem of smallrange fluctuation near the steady state point are the current research hotspots [5-7]. It not only needs to improve the control algorithm, the design methods of the observer, and the estimation method of model uncertainty $[8,9]$ but also 
needs to improve the model to expand the application range of the linear model. The purpose of developing the application range of the linear model is to make the model more accurate within a wide range of state parameters. In the controller design process, the influence of uncertainty has been considered in many types of research. In these researches, the upper bound of weight, the influence scope of parameter uncertainty, is added into the control algorithm. The analysis of influencing parameter uncertainty on the application range of the linear model is nearly blank in the aero engine modeling, and a few people studied it.

We focus on the identification model of the aero engine, which has both a broad application range and a simple form. The identification model of an aero engine can be obtained easily. The identification method on the combination of the least squares with a nonlinear filtering method is developed by Michael and Farrar, which is used in the model identification of F100 aero engine during the early stage of modeling [10]. The multivariable instrumental variable/approximate maximum likelihood method of recursive time-series analysis, proposed by Merrill, is used to identify the multivariable (four inputs-three outputs) dynamics of the Pratt \& Whitney aero engine [11]. Torres et al. [12] attempted to identify the dynamic of the gas turbine engine offline, mainly at steady states with stochastic signals. Arkov et al. [13] focused on real-time identification for transient operations and concluded that an engine system could be averaged to a timeinvariant first- or second-order transfer function by the extended recursive least squares [13]. The tracking speed and accuracy for the recursive least squares could be improved with a different design of forgetting factors. The effect of using a forgetting factor was to shift the estimating average toward the most recent data, such as that in the work of Paleologu et al. [14]. Li et al. [15] have investigated classic and modified recursive least squares algorithms for online dynamic identification of gas turbine engines. It seemed that the recursive least squares algorithm is well known for tracking dynamic systems, which is an effective conventional method of aero engine model identification. However, considering the parameter uncertainty of the identification model by recursive least squares is difficult. An identification method for the aero engine is proposed by this paper which can evaluate the parameter uncertainty of the aero engine model. Additionally, this method deals with the identification of the model by solving an optimization problem. The aero engine model may have an extensive application range, considering the parameter uncertainty of the model.

In this paper, the identification method for the aero engine parameter uncertain model is proposed. This method can identify a linear model involving model parameter uncertainty by solving the optimization problem, which is detailed in Section 2. The DGEN380 aero engine [16] is regarded as an object. The parameter uncertain model of DGEN380 is identified by DGEN380 experimental data in Sections 3.1 and 3.2. The analysis of the identification of the DGEN380 model is given in Section 3.3. Meanwhile, the parameters, VDD and VE, are defined for the error analysis of the parameter uncertain model, which is stated in Section 3.4. An example of comparing a typical least squares algorithm and the identification method for aero engine parameter uncertain model is given in Section 3.5. Section 4 is the conclusion of this paper.

\section{The Identification Method for the Parameter Uncertain Model of Aero Engine}

The aero engine model can be formulated as follows [17]:

$$
\begin{aligned}
\dot{\mathbf{x}}^{\mathrm{abs}} & =f\left(\mathbf{x}^{\mathrm{abs}}, \mathbf{u}^{\mathrm{abs}}, H, \mathrm{Ma}\right), \\
\mathbf{y}^{\mathrm{abs}} & =g\left(\mathbf{x}^{\mathrm{abs}}, \mathbf{u}^{\mathrm{abs}}, H, \mathrm{Ma}\right),
\end{aligned}
$$

where $\mathbf{x}^{\text {abs }}$ represents the aero engine state vector which concludes shaft speed, temperature, and pressure; $\mathbf{u}^{\text {abs }}$ represents the aero engine input vector, including throttle angle corresponding to the fuel flow, the nozzle area, VSV (variable stator vanes), and VBV (variable bleed valve); $\mathbf{y}^{\text {abs }}$ represents the aero engine output vector. $H$ is the flight altitude; $\mathrm{Ma}$ is flight Mach. $f$ and $g$ serve as the nonlinear functions of aero engine state variables which are vector functions of real values. The Taylor expansion is used to linearize equation (1) at a particular steady state point, by which the linear model of aero engine is obtained. This model can be represented by as follows:

$$
\begin{aligned}
& \dot{\mathbf{x}}=\mathbf{A x}+\mathbf{B u}, \\
& \mathbf{y}=\mathbf{C x}+\mathbf{D u},
\end{aligned}
$$

where $\mathbf{x}$ represents the state deviation vector of $n$ dimension, u represents the deviation vector of $m$ dimension, and y represents the output deviation vector of one dimension. These deviation variables can be described as follows:

$$
\begin{aligned}
& \mathbf{x}=\mathbf{x}^{\mathrm{abs}}-\mathbf{x}^{\mathrm{sta}}, \\
& \mathbf{u}=\mathbf{u}^{\mathrm{abs}}-\mathbf{u}^{\mathrm{sta}}, \\
& \mathbf{y}=\mathbf{y}^{\mathrm{abs}}-\mathbf{y}^{\mathrm{sta}},
\end{aligned}
$$

where $\mathbf{x}^{\text {sta }}$ represents the aero engine steady state vector, $\mathbf{u}^{\text {sta }}$ represents the aero engine steady input vector, and $\mathbf{y}^{\text {sta }}$ represents the aero engine steady output vector. And the steady variables are used to normalize the deviation variable in this research.

A steady state point of the aero engine is selected. The input pulse signal is set, which is the orthogonal vector. The matrixes $\mathbf{A}$ and $\mathbf{B}$ in equation (2) are estimated according to output response.

The static model matrix is discussed first $(\dot{\mathbf{x}}=0)$. The state of the aero engine is steady. The state deviation vector and the input deviation vector are formulated as follows:

$$
\mathbf{x}=\mathbf{G u} .
$$

The columns of matrix $\mathbf{G}$ could be confirmed by the state response vector of a given input signal $\mathbf{u}$. 
When $\dot{\mathbf{x}} \neq 0$, the state of the aero engine is dynamic. There is an assumption that matrix $\mathbf{A}$ has $n$ eigenvalues, $\lambda_{1}, \lambda_{2}, \cdots, \lambda_{n}$, and $n$ corresponding eigenvectors, $\mathbf{v}_{1}, \mathbf{v}_{2}$, $\ldots, \mathbf{v}_{n}$. Matrix $\mathbf{A}$ has $m$ repeated eigenvalue, corresponding to $m$ linearly independent eigenvectors. Matrix $\mathbf{A}$ is diagonalized similarly, and equation (5) can be obtained.

$$
\mathbf{A}=\mathbf{T} \mathbf{\Lambda} \mathbf{T}^{-1},
$$

where $\mathbf{T}=\left[\mathbf{v}_{1}, \mathbf{v}_{2}, \cdots, \mathbf{v}_{n}\right], \boldsymbol{\Lambda}=\operatorname{diag}\left(\lambda_{1}, \lambda_{2}, \cdots, \lambda_{n}\right)$.

The estimate of Matrix A means solving the programming problem $\left(\lambda_{1}, \lambda_{2}, \cdots, \lambda_{n}\right.$ and $\left.\mathbf{v}_{1}, \mathbf{v}_{2}, \ldots, \mathbf{v}_{n}\right)$

$$
\begin{aligned}
\min & \left\{\sum_{k=1}^{N}\left[{ }^{L} x(k)-{ }^{N L} x(k)\right]{ }^{T} \cdot \mathbf{W}(k) \cdot\left[{ }^{L} x(k)-{ }^{N L} x(k)\right] \mid{ }^{L} x(k)\right\} \\
& =\min \left\{\mathbf{e}^{\mathrm{T} \Lambda \mathbf{T}^{-1} \Delta t L} x(k-1)+\left(\mathbf{I}-\mathbf{e}^{\mathrm{T} \Lambda \mathbf{T}^{-1} \Delta t}\right) \cdot \mathbf{G}^{N L} \mathbf{u}(k-1)\right\},
\end{aligned}
$$

where ${ }^{N L} x(k)$ represents the discrete values of open loop state response of the nonlinear system; ${ }^{L} x(k)$ represents equation (2) corresponding to distinct values of state response of the linear system, accordingly, ${ }^{N L} x(0)={ }^{L} x(0)$; ${ }^{N L} \mathbf{u}(k)$ represents the input vector of the nonlinear system, which is orthogonal. $k=0,1, \cdots, N$, corresponding to $t_{0}, t_{1}$, $\cdots, t_{N}$; and $\Delta t$ represents the sampling time. In equation (6), $\mathbf{W}(k)$ represents the weight matrix, $\mathbf{W}(k)=\mathbf{W}^{T}(k)$, which is chosen to make the differences between nonlinear and linear model time responses more similar for all state variables.

The coefficient matrixes, $\mathbf{A}$ and $\mathbf{B}$, of the linear model are computed using equation (6). Furthermore, the influence of model parameter uncertainty is considered. Matrix $\tilde{\mathbf{A}}$ represents matrix $\mathbf{A}$ containing parameter uncertainty. Matrix $\tilde{\Lambda}$ represents diagonal matrix $\Lambda$ including uncertainty of eigenvalues. If Matrix $\tilde{\mathbf{A}}$ may be undiagonalized, Matrix $\tilde{\Lambda}$ represents Jordan matrix $\boldsymbol{\Lambda}$ including uncertainty of eigenvalues.
Equation (5) can be formulated as follows:

$$
\tilde{\mathbf{A}}=\mathbf{T} \tilde{\mathbf{\Lambda}} \mathbf{T}^{-1},
$$

where

$$
\begin{gathered}
\tilde{\lambda}_{i} \in\left[\tilde{\lambda}_{i}^{\min }, \tilde{\lambda}_{i}^{\max }\right], \quad i \in I_{R}, \\
\operatorname{Re}\left\{\tilde{\lambda}_{i}\right\} \in\left[\operatorname{Re}\left\{\tilde{\lambda}_{i}\right\}^{\min }, \operatorname{Re}\left\{\tilde{\lambda}_{i}\right\}^{\max }\right], \quad i \in I_{C}, \\
\operatorname{Im}\left\{\tilde{\lambda}_{i}\right\} \in\left[\operatorname{Im}\left\{\tilde{\lambda}_{i}\right\}^{\min }, \operatorname{Im}\left\{\tilde{\lambda}_{i}\right\}^{\max }\right], \quad i \in I_{C} .
\end{gathered}
$$

$I_{R}$ means a set of subscript indexes of real characteristic roots and $I_{C}$ means a set of subscript indexes of complex characteristic roots. For each of these complex characteristic roots, there is a complex conjugate one.

The suboptimal estimation of the range of the real parts and the imaginary parts of the eigenvalues of matrix $\tilde{\mathbf{A}}$ means that the programming problem is solved again.

$$
\begin{gathered}
\tilde{\lambda}_{i}^{\min }, \tilde{\lambda}_{i}^{\max }, \quad i \in I_{R}, \\
\operatorname{Re}\left\{\tilde{\lambda}_{i}\right\}^{\min }, \operatorname{Re}\left\{\tilde{\lambda}_{i}\right\}^{\max }, \quad i \in I_{C}, \\
\operatorname{Im}\left\{\tilde{\lambda}_{i}\right\}^{\min }, \operatorname{Im}\left\{\tilde{\lambda}_{i}\right\}^{\max }, \quad i \in I_{C}, \\
\min \left\{\sum_{k=1}^{N}\left[{ }^{L U} x(k)-{ }^{N L} x(k)\right]^{T} \cdot \mathbf{W}(k) \cdot\left[{ }^{L U} x(k)-{ }^{N L} x(k)\right]\right\},
\end{gathered}
$$

where ${ }^{N L} x(0)={ }^{L U} x(0), k=0,1, \cdots, N,{ }^{L U} x(k)$ represents the discrete values of uncertain system state response in equation (2). The calculation of the objective function in equation (9) needs $N$ multiple nonlinear programming problems.

$$
\begin{aligned}
& { }^{L U} x(k), \\
& \min _{M}\left\{\left.\sum_{k=1}^{N}\left[{ }^{L U} x(k)-{ }^{N L} x(k)\right]^{T} \cdot \mathbf{W}(k) \cdot\left[{ }^{L U} x(k)-{ }^{N L} x(k)\right]\right|^{L U} x(k)\right\} \\
& =\min _{M}\left\{\mathbf{e}^{\mathrm{T} \tilde{\mathbf{\Lambda}}(k-1) \mathbf{T}^{-1} \Delta t L U} x(k-1)+\left(\mathbf{I}-\mathbf{e}^{\mathrm{T} \tilde{\mathbf{\Lambda}}(k-1) \mathbf{T}^{-1} \Delta t}\right) \cdot \mathbf{G}^{N L} \mathbf{u}(k-1)\right\}, \\
& M=\left\{\tilde{\lambda}_{i}(k-1) \in\left[\tilde{\lambda}_{i}^{\min }, \tilde{\lambda}_{i}^{\max }\right], \quad i \in I_{R},\right. \\
& \operatorname{Re}\left\{\tilde{\lambda}_{i}(k-1)\right\} \in\left[\operatorname{Re}\left\{\tilde{\lambda}_{i}\right\}^{\min }, \operatorname{Re}\left\{\tilde{\lambda}_{i}\right\}^{\max }\right], \quad i \in I_{C} \\
& \left.\operatorname{Im}\left\{\tilde{\lambda}_{i}(k-1)\right\} \in\left[\operatorname{Im}\left\{\tilde{\lambda}_{i}\right\}^{\min }, \operatorname{Im}\left\{\tilde{\lambda}_{i}\right\}^{\max }\right], \quad i \in I_{C},\right\}
\end{aligned}
$$


where

$$
\begin{aligned}
\tilde{\Lambda}(k-1) & =\operatorname{diag}\left\{\tilde{\lambda}_{1}(k-1), \tilde{\lambda}_{2}(k-1), \cdots, \tilde{\lambda}_{n}(k-1)\right\}, \\
\mathbf{W}(k) & =\mathbf{W}^{T}(k)>0 .
\end{aligned}
$$

The weight matrix $\mathbf{W}(k)$ is used to reduce the difference between the state response vector of the nonlinear system and the state response vector of the linear system containing uncertainty.

The solution of equation (6) is an initial condition to solve programming problems. The optimal solution of matrix $\mathbf{A}$ and the eigenvalues of matrix $\mathbf{A}$ are used as the initial condition to estimate their uncertain range. The parameter uncertain model can be gotten by the calculation of programming problems in equations (9) and (10). Equations (9) and (10) are solvable, which means optimization problem has a nonempty solution set. This problem is proved in Ref. [18].

\section{The Analysis of Identification of DGEN380 Model}

This section is about the application of the identification method of the parameter uncertain model, which is used to identify the DGEN380 aero engine model. The start-up process and shutdown process are ignored. The maximum continuous power point is selected as a steady state in the model identification process. The flight altitude is $3048 \mathrm{~m}$, $\mathrm{Ma}$ is 0.338 , and the throttle angle is $74 \%$ at this state point.

The engine state vector $\mathbf{x}$ includes the rotation speed of the high-pressure rotor, the rotation speed of the lowpressure rotor, the exit pressure of the high-pressure compressor, and the exit pressure and temperature of the low-pressure turbine. The engine input variable is fuel flow. As shown in equation (12), $n=5$ and $m=1$ can be known.

The state variable $N_{1}$ represents the speed of the lowpressure shaft, and the state variable $N_{2}$ is the speed of the high-pressure shaft. The state variable $P_{3}$ represents the import pressure of the combustor. The state variable $P_{5}$ represents the export temperature of the low-pressure turbine, and the state variable $T_{5}$ represents the export temperature of the low-pressure turbine. The input variable $W_{f}$ is the fuel flow of DGEN380.

$$
\begin{aligned}
& \mathbf{x}^{\mathrm{abs}}=\left[N_{1}, N_{2}, P_{3}, P_{5}, T_{5}\right]^{T}, \\
& \mathbf{u}^{\mathrm{abs}}=\left[W_{f}\right] .
\end{aligned}
$$

A parameter uncertainty model will be obtained. Moreover, a comparison is implemented by experimental data, the parameter uncertainty model, and another DGEN380 linear model. Then an error analysis is exerted by parameters VDD and VE.

3.1. DGEN380 Engine Experimental Device. The experimental device contains a test bench and a control desktop. The

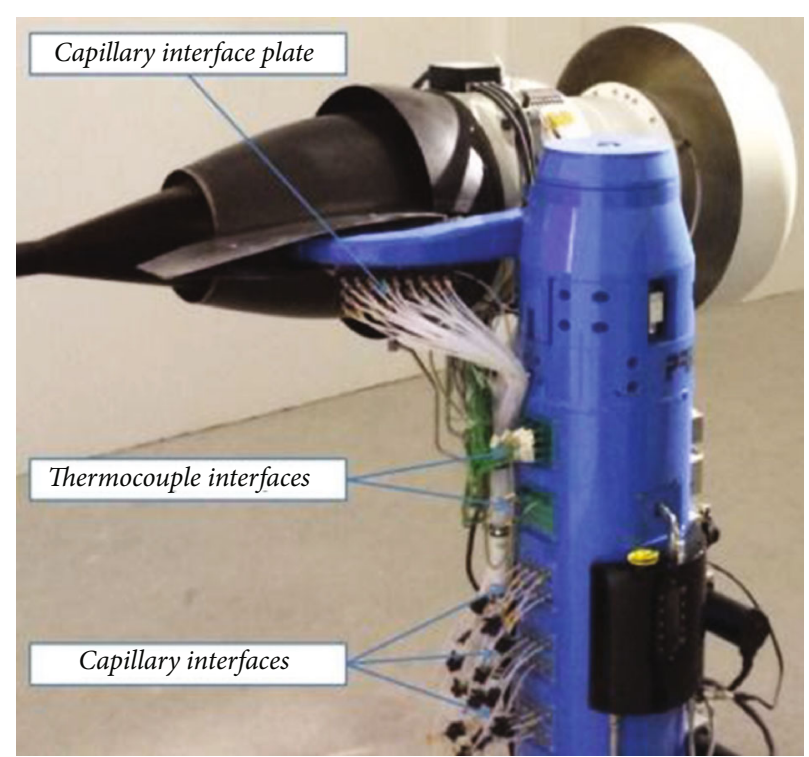

FIgURE 1: DGEN380 test bench.

test bench and control desktop are shown in Figures 1 and 2, respectively. The test bench is composed of an DGEN380 engine, measuring transducers, sensor baronesses, and integrators (a blue pillar in Figure 1). The main functions of the test bench are engine operating, parameter measurement, and signal transmission. The control desktop is composed of a power lever, a FADEC controller of DGEN380, and a video screen. The primary functions of the control desktop are to control the aero engine state by the power lever, to show engine state and parameter values, and to monitor the bench state.

The rotor speed of DGEN380 engine is measured by a magnetoelectric tachometric transducer. $N_{1}$ speed sensor locates fan casing. $N_{2}$ speed sensor determines the motor starter. $P_{5}$ is measured by the resonant pressure sensor. The outlet air of the low-pressure turbine is lead out by a drainage tube, which flows to the pressure sensor in the testing signal concentrator. $T_{5}$ is measured by the thermocouple transducer. Measured values are transmitted to the testing signal concentrator by a wiring harness. The detailed description of the experimental device may be referred to [16].

In the experiment, the parameters are set: $N=1000$ and $\Delta t=0.025 \mathrm{~s}$.

\subsection{The Results of Identification of DGEN380 Parameter} Uncertain Model. The test bench exerts the experiment. When the DGEN380 is stable at the maximum continuous power point (PLA $=74 \%$ ), the pulse-response experiments are performed.

The experimental data can be used to solve equation (6). By this method, the linear model of the DGEN380 engine at the maximum continuous thrust operating point is obtained. The eigenvalues of this model are shown in Table 1. Matrix A contains five eigenvalues, among which there are a pair of conjugate complex features. This model is an initial condition of the suboptimal estimation. 


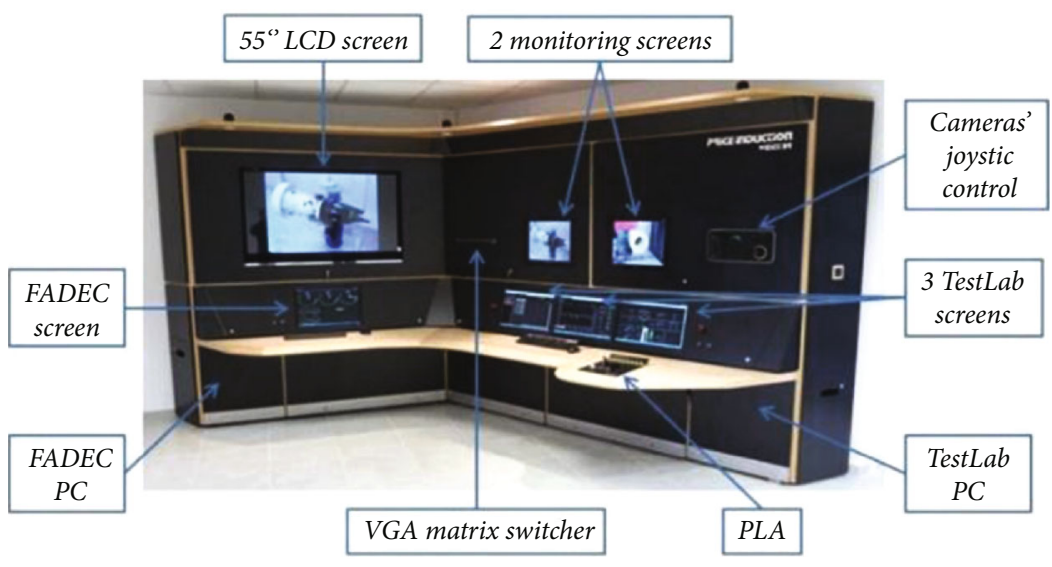

Figure 2: General view of the control desktop.

TABLE 1: The eigenvalue of the DGEN380 linear model.

\begin{tabular}{lccccc}
\hline Eigenvalue $\lambda_{i}$ & $\lambda_{1}$ & $\lambda_{2}$ & $\lambda_{3}$ & $\lambda_{4}$ & $\lambda_{5}$ \\
\hline Value & -0.852 & -5.605 & $-14.789+0.035 j$ & $-14.789-0.035 j$ & -10.210 \\
\hline
\end{tabular}

According to Table 1, the suboptimal estimations (equations (9) and (10)) are solved. By this way, the DGEN380 parameter uncertain model can be gotten at the maximum continuous power operating point. The upper and lower bounds of the eigenvalues of the parameter uncertain model are shown in Table 2.

\subsection{Comparison between Simulation Results and Experiment} Results. The purpose of the comparison is (1) to reveal the error between the state value computed by the parameter uncertain model and experimental value and (2) to compare the model behavior between the parameter uncertainty model and a linear model. This linear model of DGEN380 is selected for comparison that is built at the maximum continuous power point by NASA Glenn Research Center [17]. This model is often used for DGEN380 controller design and diagnosis algorithm design which has universality because of the high accuracy.

The experiment is performed on the test bench. When the DGEN380 is stable at the maximum continuous power point $(\mathrm{PLA}=74 \%)$, the power lever is pushed from PLA = $74 \%$ to PLA $=64 \%$ suddenly. The experiment may be recorded. Then, the simulation is implemented by the parameter uncertain model and the linear model of NASA. The comparison results are shown in Figures 3-7. It can be seen from the figures that the trend of each component of the state vector $\mathbf{x}$ is decreasing. The state value decreases from the steady state value to another value corresponding to $\mathrm{PLA}=64 \%$. There is a small oscillation in this dying process.

Compared with those of the linear model, the calculation results of the parameter uncertain model agree better with the experimental curve. When $\triangle \mathrm{PLA}=10 \%$, the trend of the state variables obtained by the linear model is consistent with the experimental data. However, there are apparent
TABLE 2: The eigenvalue of the DGEN380 parameter uncertain model.

\begin{tabular}{lcc}
\hline Eigenvalue $\tilde{\lambda}_{i}$ & Lower bound (min) & Upper bound (max) \\
\hline$\tilde{\lambda}_{1}$ & -2.175 & -0.055 \\
$\tilde{\lambda}_{2}$ & -7.520 & -2.186 \\
$\operatorname{Re}\left\{\tilde{\lambda}_{3}\right\}$ & -65.125 & -8.565 \\
$\operatorname{Im}\left\{\tilde{\lambda}_{3}\right\}$ & 0.042 & 0.068 \\
$\operatorname{Re}\left\{\tilde{\lambda}_{4}\right\}$ & -65.125 & -8.565 \\
$\operatorname{Im}\left\{\tilde{\lambda}_{4}\right\}$ & -0.068 & -0.042 \\
$\tilde{\lambda}_{5}$ & -22.055 & -6.311 \\
\hline
\end{tabular}

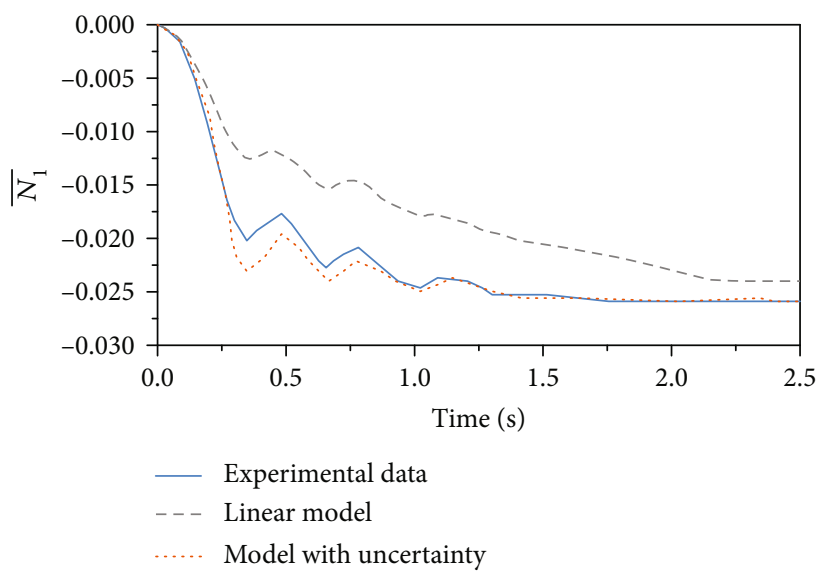

Figure 3: The response curve of low-pressure rotor. 


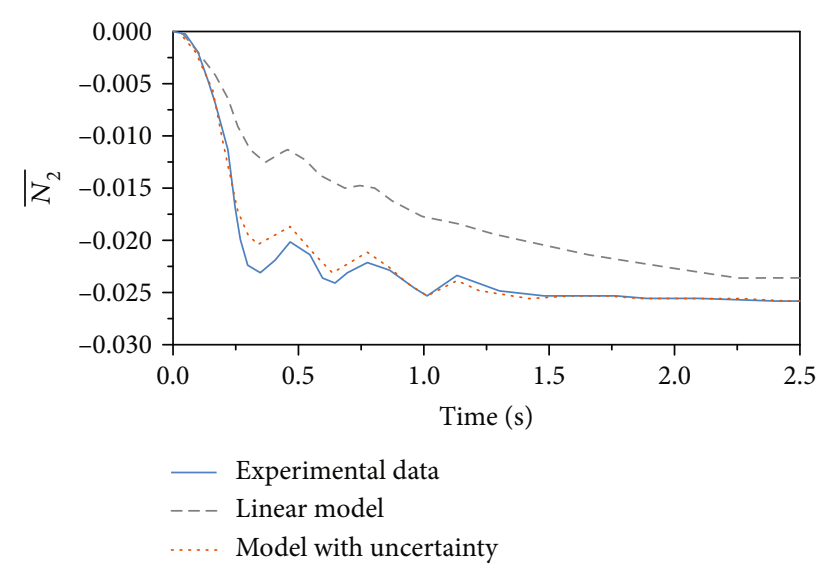

FIgURE 4: The response curve of high-pressure rotor.

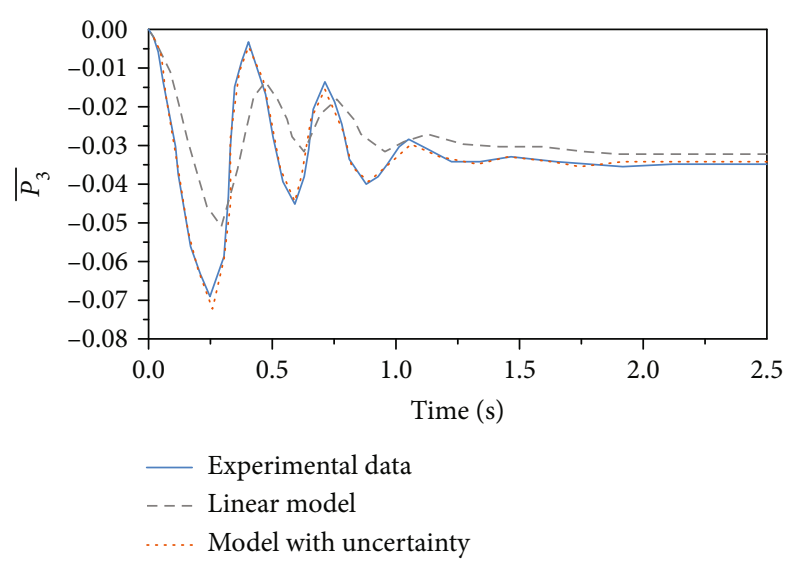

Figure 5: The response curve of the exit of high-pressure compressor.

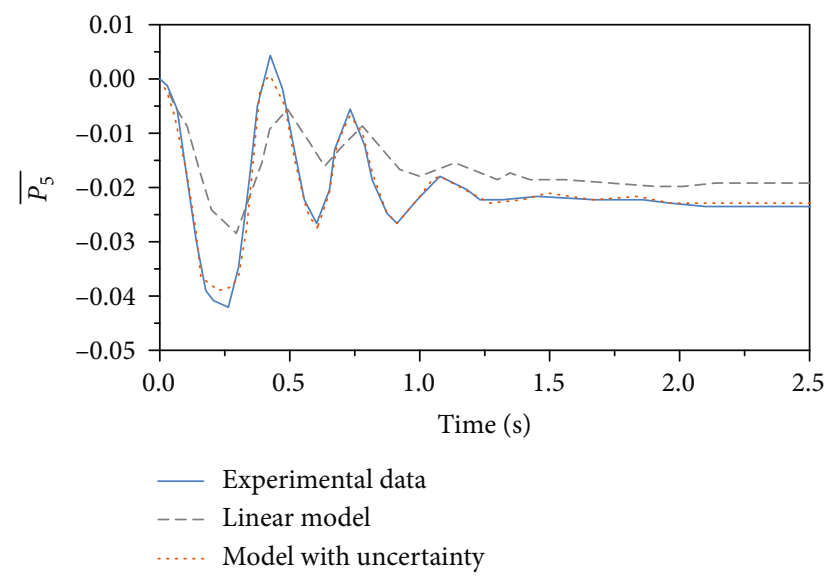

FIGURE 6: The response curve of the exit of high-pressure turbine.

differences between the linear model curve path and the experimental curve path. The response time and static value of the linear model are not the same as experimental data. The simulating results of the parameter uncertain model

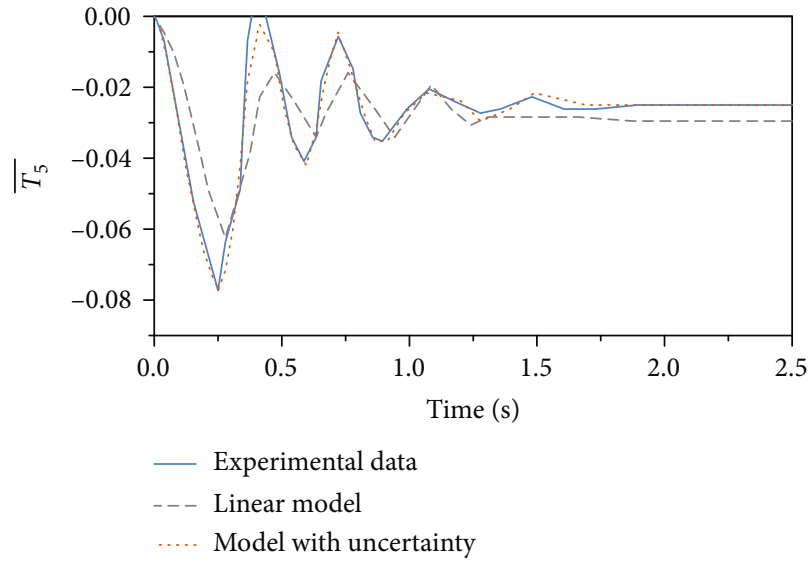

FIgURE 7: The response curve of the exit of low-pressure turbine.

are identical with the data obtained from the experiment. The calculation results of the parameter uncertain model can reproduce the experimental results with small errors.

The value of state vector change would occur when the aero engine state moves from one state to another state. There is not an approximately linear correlation between parameters of the aero engine when the deviation of the aero engine state from the steady state is rather significant. At this time, the $f^{\prime}$ in formula (1) cannot be approximately shown by the slope of the linear model. There is a large difference between $f^{\prime}$ and the slope. Therefore, the phenomenon of model mismatch appears when the aero engine state deviates from the steady state largely. However, the engine parameter uncertain model, to estimate the variation range of the coefficient matrix eigenvalues, considers the impact of model parameter uncertainty, which can offset the influence of the model mismatch in a field.

Comparing the calculation results of the linear model with the results of the experiment, the error is more significant between the calculation results of the linear model and experiment data due to linear model mismatch during when the power lever deviates by $10 \%$. Comparing the calculation results of the parameter uncertain model with the results of the experiment, the calculation results of the engine parameter uncertain model agree better with experimental results.

3.4. The Error Analysis of Parameter Uncertain Model. This section analyzes the applied range of the DGEN380 parameter uncertain model in the maximum continuous power point.

There are two parameters defined by equations (13) and (14).

$$
\mathrm{VDD}=\sqrt{\sum_{k=1}^{N}\left[{ }^{L} \bar{x}(k)-{ }^{\operatorname{Exp}} \bar{x}(k)\right]^{T} \cdot \mathbf{W}(k) \cdot\left[{ }^{L} \bar{x}(k)-{ }^{\operatorname{Exp}} \bar{x}(k)\right]},
$$

$$
\mathrm{VE}=\max \left\{\left|{ }^{\mathrm{LU}} \bar{x}(k)-{ }^{\operatorname{Exp}} \bar{x}(k)\right|\right\}
$$




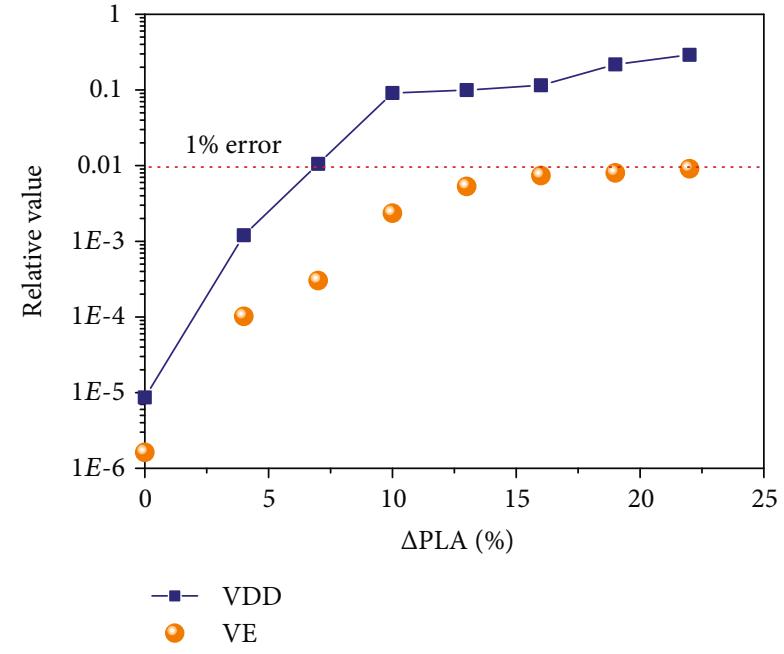

FIgURE 8: The relation graph between PLA and VDD, SD.

$$
\begin{aligned}
& \exists \delta>0, \\
& \text { s.t. }|\mathrm{VDD}-0|<\delta,
\end{aligned}
$$

where $\bar{x}$ represents the relative value, superscript Exp represents the experiment data, and $\mathbf{W}$ represents the weight matrix. VDD reflects the deviation between the real engine state and operating state based on the difference between the calculated results of the linear model and experimental data. This linear model, built by NASA, has a full applied range. The smaller the VDD figured, the less distance between the linear model and real state of aero engine. When the engine is at the operating point, VDD meets formula (15). The maximum relative error of the parameter uncertain model can be calculated by VE.

The maximum continuous power point (PLA $=74 \%)$ is regarded as the initial state of DGEN380. VDD and VE are calculated based on the simulated data and experimental data, respectively, when $\triangle \mathrm{PLA}$ is $4 \%, 7 \%, 10 \%, 13 \%, 16 \%$, $19 \%$, and $22 \%$. Setting $\mathbf{W}(k)=\mathbf{I}_{5 * 5}$, the calculation results are shown in Figure 8. The red dot line, in Figure 8, indicates the $1 \%$ deviation position.

As the $\triangle \mathrm{PLA}$ continues to increase, the VDD curve rises significantly. It manifests that the mismatch of the linear model is more significant and more substantial as the $\Delta$ PLA increases. When $\triangle \mathrm{PLA}=7 \%$, the cumulative error of the state vector $\mathbf{x}$ calculated by the linear model reaches $1 \%$; when $\triangle \mathrm{PLA}>10 \%$, the cumulative error of the state vector $\mathbf{x}$ exceeds $10 \%$. The farther the PLA is from the steady state point, the more seriously the linear model mismatches at the operating position.

As the $\triangle \mathrm{PLA}$ continues to increase, the VE value gradually increases. The maximum error between the calculation results of the parameter uncertain model and experimental data gradually increases. However, the maximum error values increase that is no more than $1 \%$ slowly. The eight orange dots, in Figure 8, are all below the, 1\% error, red dot line. When $\triangle \mathrm{PLA}<22 \%$, the deviation between the calculation results of the parameter uncertain model and engine real state is smaller, which can simulate the actual engine state in $1 \%$ error range.

3.5. A Simple Example of Comparison of Two Identification Methods' Performance. The identification method for the parameter uncertain model is proposed. Meanwhile, the parameter uncertain model, obtained by this method, can keep a small error level in a broad range of engine state deviation from a particular state, from Section 3.3.

The least squares algorithm is well known for model identification, which is an effective conventional method of aero engine model identification. The recursive least squares (RLS) method is a typical representative of the least squares algorithm. The recursive least squares method, proposed by Li et al. [15], is an advanced identification method for an aero engine model. The aero engine identification model, obtained by the RLS algorithm, has an excellent performance. So the RLS algorithm and the identification method for the parameter uncertain model are both used in aero engine model identification in this chapter.

The component level model of JT9D of Pratt \& Whitney, a civil high bypass aero engine, is used for the example of this chapter which is established by NASA Glenn Research Center, based on the T-MATS Module of MATLAB/Simulink. The cruise power point is selected as a steady state in the model identification process. The flight altitude is $10200 \mathrm{~m}$, $\mathrm{Ma}$ is 0.77 , and the throttle angle is $54 \%$ at this state point. The engine state vector $\mathbf{x}$ includes the rotation speed of the high-pressure rotor $\left(N_{2}\right)$, the rotation speed of the lowpressure rotor $\left(N_{1}\right)$, the exit pressure of the high-pressure compressor $\left(P_{3}\right)$, and the exit pressure and temperature of the high-pressure turbine $\left(T_{45}\right)$. The engine input variable is fuel flow $\left(W_{f}\right)$. All of these variables are necessary for normalization processing.

The experiment is performed on the component level model of JT9D. An input pulse signal is set. The response data of the component level model is used for model identification. Two identification models can be obtained. One is the parameter uncertain model. Another is the RLS algorithm model.

To compare these models, different $\triangle \mathrm{PLA}$ values are set. When the component level model is stable at the cruise power point $(\mathrm{PLA}=54 \%),(1)$ the power lever is pushed from $\mathrm{PLA}=54 \%$ to $\mathrm{PLA}=59 \%$ suddenly $(\Delta \mathrm{PLA}=5 \%),(2)$ the power lever is pushed from PLA $=54 \%$ to PLA $=64 \%$ suddenly $(\triangle \mathrm{PLA}=10 \%)$, and $(3)$ the power lever is pushed from $\mathrm{PLA}=54 \%$ to $\mathrm{PLA}=69 \%$ suddenly $(\triangle \mathrm{PLA}=15 \%)$. The experimental may be recorded. Then, the simulation is implemented by the parameter uncertain model and the RLS algorithm model. The comparison results are shown in Figure 9. Compared with the RLS algorithm mode, the calculation results of the parameter uncertain model agree better with the curve of the component level model.

When $\triangle \mathrm{PLA}=5 \%$, the curves of the two identification models agree better with the curve of the component level model. When $\triangle \mathrm{PLA}>5 \%$, the difference between the RLS algorithm model and component level model is more and more evident. The deviation between the parameter uncertain 

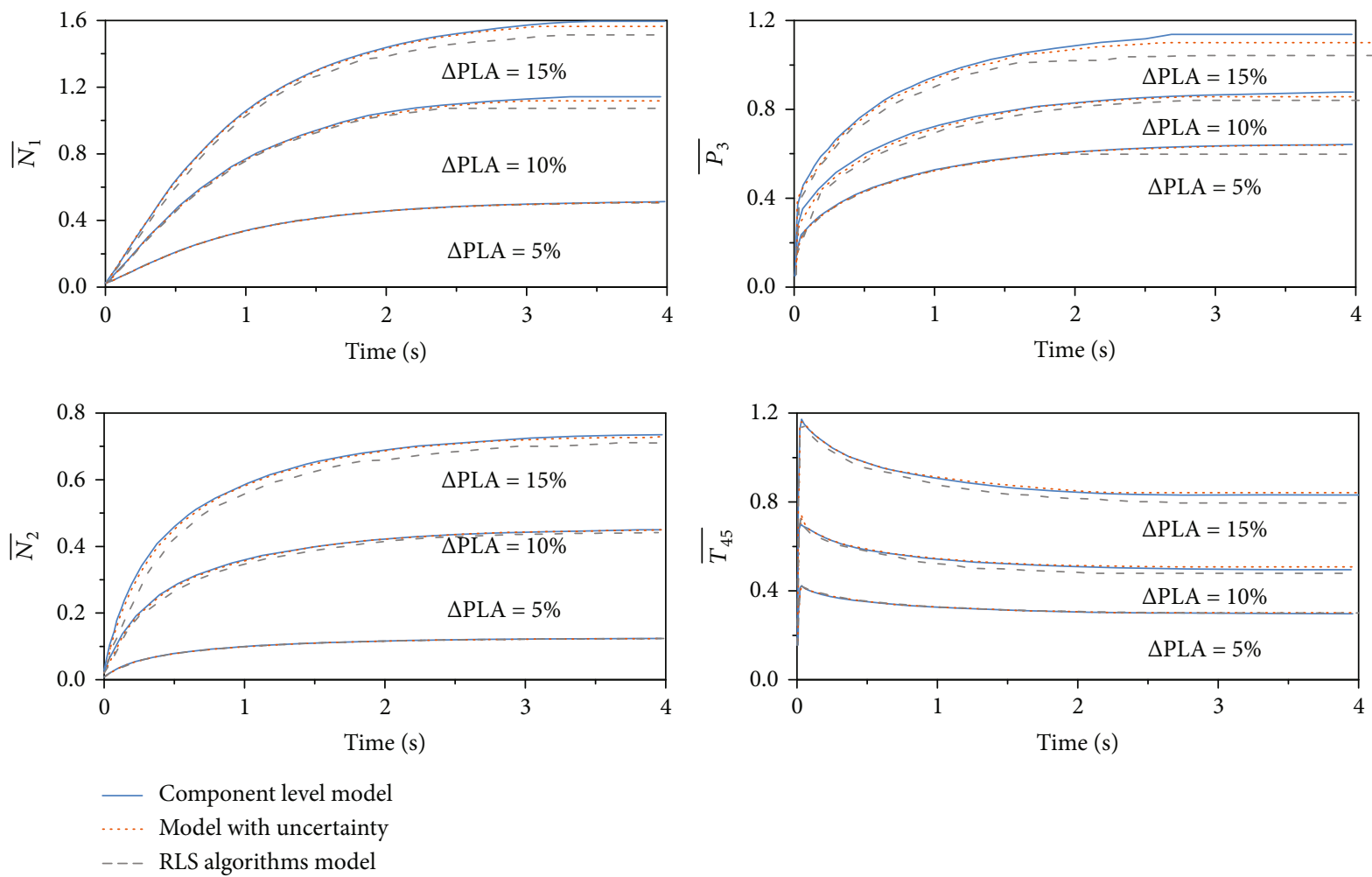

Figure 9: The response curve of two models.

TABLE 3: The value of VDD and VE $(\triangle \mathrm{PLA}=15 \%)$.

\begin{tabular}{lcc}
\hline & RLS algorithm model & Parameter uncertain model \\
\hline VDD & 0.15 & 0.073 \\
VE & 0.022 & 0.0064 \\
\hline
\end{tabular}

model and component level model is tiny. When $\triangle \mathrm{PLA}=$ $15 \%$, Table 3 describes the value of VDD and VE. From this table, both VDD and VE values of the RLS algorithm model are more significant than those of another model. Though the performance of the RLS algorithm model may be useful, the performance of the parameter uncertain model is much better, especially at $\triangle \mathrm{PLA}=15 \%$.

The RLS method is a suitable method which is a conventional method in model identification of aero engine. But the application range of the model identified by RLS is small. The identification method for the parameter uncertain model can obtain a linear model by solving the optimization problem. This method can consider, if it is essential, the parameter uncertainty of the model, which leads to the parameter uncertain model having a broad application area.

\section{Conclusions}

In this paper, an identification method for the aero engine parameter uncertain model is proposed. This method used the programming method to deal with the aero engine model identification problem which considers the influence of model parameter uncertainty. This method enables obtaining the bounds for real and imaginary parts of uncertain matrix eigenvalues of a parameter uncertain model from aero engine experiment data. Its modeling errors meet the current control requirements. The method uses nonlinear programming and can consequently consider any constraints for the estimated bounds for real and imaginary parts of uncertain matrix eigenvalues.

Based on the DGEN380 aero engine experimental data, the parameter uncertain model of the DGEN380 engine at the maximum continuous power point is identified. The differences between the parameter uncertain model and the linear model of DGEN380 used to design controller extensively can be compared by calculating. The comparison results indicate that the parameter uncertain model can simulate the DGEN380 aero engine real state at the maximum continuous power point when $\triangle \mathrm{PLA}=10 \%$. Then the error range of the parameter uncertain model is analyzed by the defined parameters VDD and VE. The analysis results manifest that the error of the engine parameter uncertain model increases slowly and is small, as the mismatch of the linear model is more and more serious. When $\triangle \mathrm{PLA}<22 \%$, it can simulate the real engine state in $1 \%$ error. The parameter uncertain model not only has the advantages of the simple form and fast calculation speed of the linear model but also can maintain a small error level in a broad range of engine state deviation from the operating point state. Considering the parameter uncertainty of the model is the main contribution 
of this research during the optimization process. The model is used for the design of aero engine fault diagnosis and engine control algorithm, which can expand the application range of diagnostic methods and control methods in lower error intervals.

\section{Data Availability}

The [DATA_6015270.xlsx] data used to support the findings of this study may be released upon application to the School of Mechanical Engineering of the Hebei University of Technology, which can be contacted through Dr. Shuai Liu (e-mail: caucliushuai@163.com).

\section{Conflicts of Interest}

The authors declare that they have no conflicts of interest.

\section{References}

[1] J. Csank, R. May, J. Litt, and T.-H. Guo, "Control design for a generic commercial aircraft engine," in 46th AIAA/ASME/SAE/ASEE Joint Propulsion Conference \& Exhibit, pp. 305322, Nashville, TN, USA, 2010.

[2] A. Gimelli and R. Sannino, "Thermodynamic model validation of Capstone C30 micro gas turbine," Energy Procedia, vol. 126, pp. 955-962, 2017.

[3] J. Litt, D. Frederick, and T.-H. Guo, "The case for intelligent propulsion control for fast engine response," in AIAA Infotech@Aerospace Conference, pp. 1157-1166, Seattle, WA, USA, 2009.

[4] A. Zandi Nia and R. Nagamune, "Switching gain-scheduled proportional-integral-derivative electronic throttle control for automotive engines," Journal of Dynamic Systems, Measurement, and Control, vol. 140, no. 7, article 071015, 2018.

[5] H. Richter and J. Litt, "A novel controller for gas turbine engines with aggressive limit management," in 47th AIAA/ASME/SAE/ASEE Joint Propulsion Conference \& Exhibit, pp. 1-17, San Diego, CA, USA, 2011.

[6] A. Imani and M. Montazeri-Gh, "Improvement of min-max limit protection in aircraft engine control: an LMI approach," Aerospace Science and Technology, vol. 68, pp. 214-222, 2017.

[7] S. Liu, J. Bai, Q. Wang, and W. Wang, "Tracking controller design for aero-engine based on improved multi-power reaching law of sliding mode control," International Journal of Aeronautical and Space Sciences, vol. 20, no. 3, pp. 722-731, 2019.

[8] X. Du, H. Richter, and Y. Guo, "Multivariable sliding-mode strategy with output constraints for aeroengine propulsion control," Journal of Guidance, Control, and Dynamics, vol. 39, no. 7, pp. 1631-1642, 2016.

[9] F. Lu, H. Ju, and J. Huang, "An improved extended Kalman filter with inequality constraints for gas turbine engine health monitoring," Aerospace Science and Technology, vol. 58, pp. 36-47, 2016.

[10] G. Michael and F. Farrar, Identification of multivariable gas turbine dynamics from stochastic input-output data, DTIC Document, 1975.

[11] W. Merrill, "Identification of multivariable high-performance turbofan engine dynamics from closed-loop data," Journal of Guidance, Control, and Dynamics, vol. 7, no. 6, pp. 677-683, 1984.
[12] M. P. Torres, G. Sosa, L. Amezquita-Brooks, E. Liceaga-Castro, and P. C. del Zambrano-Robledo, "Identification of the fuelthrust dynamics of a gas turbo engine," in 52nd IEEE Conference on Decision and Control, pp. 4535-4540, Florence, Italy, 2013.

[13] V. Arkov, C. Evans, P. J. Fleming et al., "System identification strategies applied to aircraft gas turbine engines," Annual Reviews in Control, vol. 24, no. 1, pp. 67-81, 2000.

[14] C. Paleologu, J. Benesty, and S. Ciochiňa, "A robust variable forgetting factor recursive least-squares algorithm for system identification," IEEE Signal Processing Letters, vol. 15, pp. 597-600, 2008.

[15] Z. Li, T. Nikolaidis, and D. Nalianda, "Recursive least squares for online dynamic identification on gas turbine engines," Journal of Guidance, Control, and Dynamics, vol. 39, no. 11, pp. 2594-2601, 2016.

[16] “Price induction DGEN engine specications," December 2015, http://www.price-induction.com/dgen-engine/.

[17] J. W. Connolly, J. Csank, A. Chicatelli, and K. Franco, "Propulsion controls modeling for a small turbofan engine," in 53rd AIAA/SAE/ASEE Joint Propulsion Conference, pp. 1-15, Atlanta, GA, 2017.

[18] H. Genceli and M. Nikolaou, "Robust stability analysis of constrained $l_{1}$-norm model predictive control," AICHE Journal, vol. 39, no. 12 , pp. 1954-1965, 1993. 


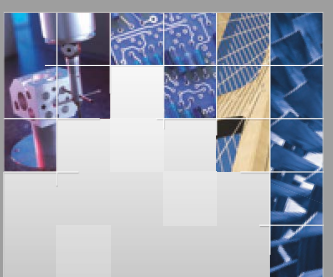

\section{Enfincering}
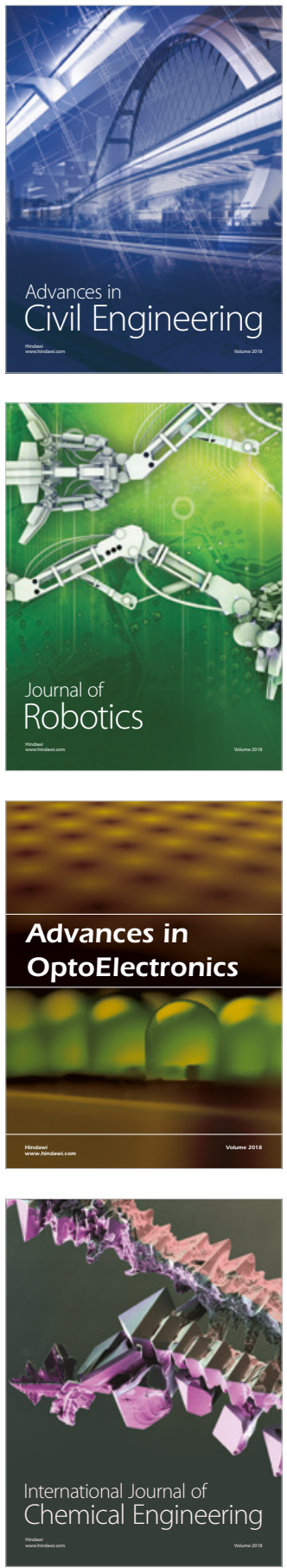

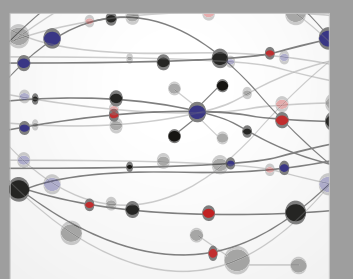

\section{Rotating \\ Machinery}

The Scientific World Journal

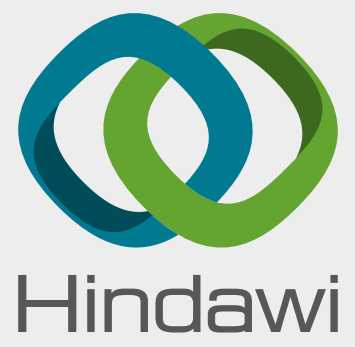

Submit your manuscripts at

www.hindawi.com
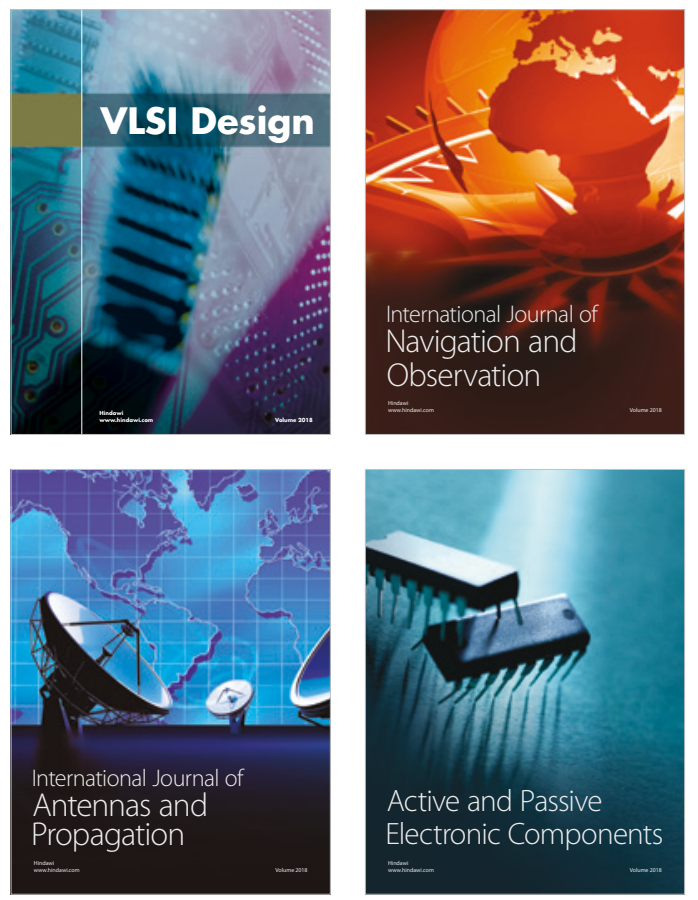
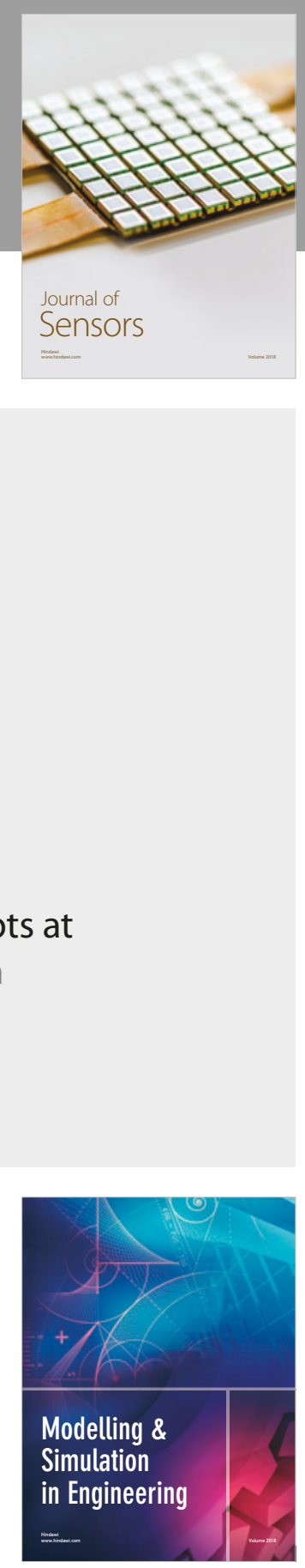

\section{Advances \\ Multimedia}
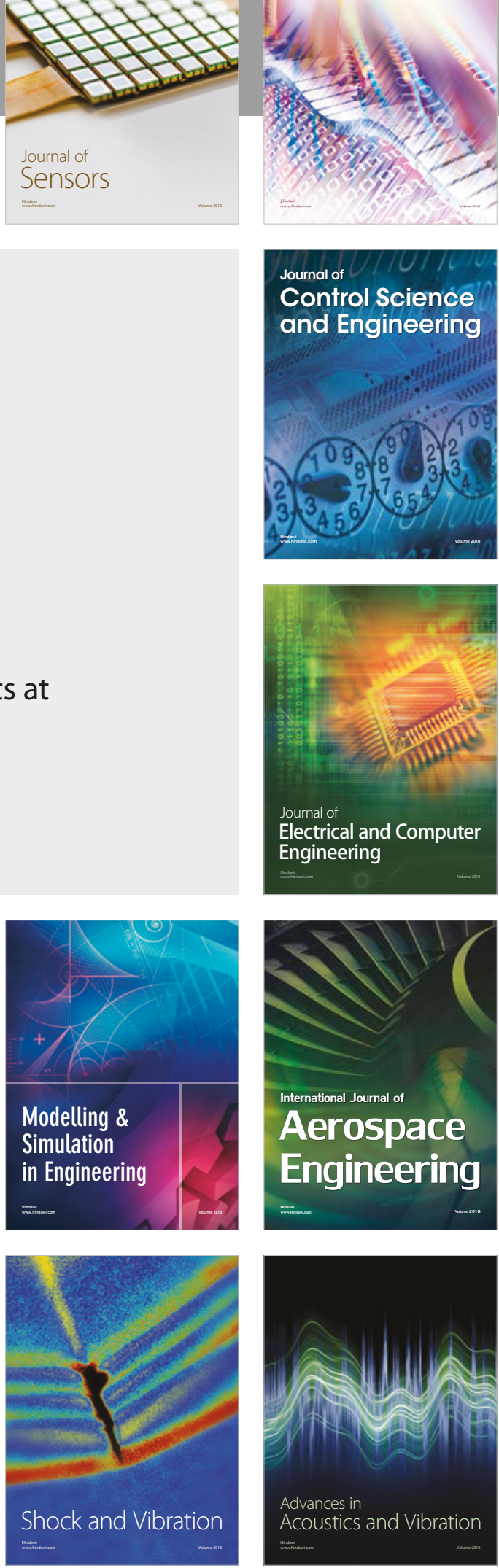